\title{
Experimenting with Infinite Groups, I
}

\author{
Gilbert Baumslag, Sean Cleary, and George Havas
}

\section{CONTENTS}

1. Introduction

2. Background

3. Experimental Design

4. Computational Results

5. Further Questions

Acknowledgments

References
2000 AMS Subject Classification: Primary 20-04, 20E26; Secondary 20F05, 20F10

Keywords: Infinite groups, parafree groups, finite quotients
A group is termed parafree if it is residually nilpotent and has the same nilpotent quotients as a given free group. Since free groups are residually nilpotent, they are parafree. Nonfree parafree groups abound and they all have many properties in common with free groups. Finitely presented parafree groups have solvable word problems, but little is known about the conjugacy and isomorphism problems. The conjugacy problem plays an important part in determining whether an automorphism is inner, which we term the inner automorphism problem. We will attack these and other problems about parafree groups experimentally, in a series of papers, of which this is the first and which is concerned with the isomorphism problem. The approach that we take here is to distinguish some parafree groups by computing the number of epimorphisms onto selected finite groups. It turns out, rather unexpectedly, that an understanding of the quotients of certain groups leads to some new results about equations in free and relatively free groups. We touch on this only lightly here but will discuss this in more depth in a future paper.

\section{INTRODUCTION}

One-relator groups form a very interesting setting for understanding a number of difficult algorithmic problems about finitely presented groups. The simplest finitely presented parafree groups are the finitely generated free groups, for which Dehn's fundamental algorithmic questions addressing the word, conjugacy, and isomorphism problems are trivially solvable. The situation is already very different in the next-simplest possible set of finitely presented parafree groups, those defined by a single relation. Magnus [Magnus 30] solved the word problem for one-relator groups in general but, despite remarkable progress in a number of cases, the general conjugacy problem for one-relator groups remains open. The even more difficult isomorphism problem for one-relator groups lies even further out of reach. Here, we consider a computational approach to the isomorphism problem for some very restricted classes of one-relator groups by studying several families of one-relator parafree groups.

(C) A K Peters, Ltd. $1058-6458 / 2004 \$ 0.50$ per page Experimental Mathematics 13:4, page 495 
Theoretical approaches have had limited success in determining when groups in these families are isomorphic, so we approach this question computationally. Our results suggest that a one-relator parafree group is determined by its finite images. More precisely, we suspect that if two one-relator parafree groups have the same set of finite images, then they are isomorphic. A slightly different but more wishful possibility is that if $G$ is finitely generated and residually finite and if $G$ has the same finite quotients as a free group, then $G$ is free. Here the hypothesis that $G$ be finitely generated is essential ([Baumslag 68a]). As a byproduct of this line of thinking we prove, not surprisingly, that if two finitely generated groups, $G$ and $H$, have the same set of finite images and if $T$ is any finite group, then the number of epimorphisms from $G$ onto $T$ is the same as the number of epimorphisms from $H$ onto $T$. This does not seem to have been noted before.

\section{BACKGROUND}

Parafree groups have many properties in common with free groups yet are not necessarily themselves free. These groups are generally very hard to distinguish from one another and are even harder to distinguish from free groups (see [Baumslag 67a, Baumslag 68a, Baumslag 68b, Baumslag 94] and [Baumslag and Cleary 05]). We begin by introducing some notation in order to define parafree groups.

Given $x$ and $y$, elements in a group $G$, we denote by $x^{y}$ the conjugate $y^{-1} x y$ of the element $x$ by the element $y$, and we denote the commutator $x^{-1} y^{-1} x y$ of $x$ and $y$ by $[x, y]$. The lower central series

$$
G=\gamma_{1}(G) \geq \gamma_{2}(G) \geq \ldots \geq \gamma_{n}(G) \geq \ldots
$$

is defined inductively by

$$
\gamma_{n+1}(G)=\operatorname{gp}\left([x, y] \mid x \in \gamma_{n}(G), y \in G\right) .
$$

$G$ is termed residually nilpotent if

$$
\bigcap_{n=1}^{\infty} \gamma_{n}(G)=1 .
$$

Equivalently, $G$ is residually nilpotent if given any nontrivial element $g \in G$, there exists a normal subgroup $N$ of $G$ such that $g \notin N$ with $G / N$ nilpotent. In general, if $\mathcal{P}$ is a property or class of groups, then $G$ is termed residually $\mathcal{P}$ if, given any nontrivial element $g \in G$, there exists a normal subgroup $N$ of $G$ such that $g \notin N$ with $G / N \in \mathcal{P}$.
We now define a group $G$ to be parafree if $G$ is residually nilpotent and there exists a free group $F$ such that $G / \gamma_{n}(G) \cong F / \gamma_{n}(F)$ for every integer $n \geq 1$. It follows that $G$ has the same nilpotent factor groups as $F$, which means that there is no way to distinguish $G$ from $F$ if we restrict our attention to nilpotent groups.

\subsection{Families of Parafree Groups}

There are now many known families of parafree groups. The primary objective of this paper is to begin to distinguish some of the members of these families from each other and from free groups.

Baumslag [Baumslag 67b] introduced a family of parafree groups which we denote $G_{i, j}$ and which is presented as

$$
G_{i, j}=\left\langle a, b, c \mid a=\left[c^{i}, a\right]\left[c^{j}, b\right]\right\rangle .
$$

Baumslag [Baumslag 94] later introduced other families which include some we denote $H_{w}$ and special cases of these denoted $H_{i, j}$, where $w$ is a word in the derived group and $i$ and $j$ are positive integers. These are presented as

$$
\begin{gathered}
H_{w}=\langle a, s, t \mid a=w[s, t]\rangle ; \\
H_{i, j}=\left\langle a, s, t \mid a=\left[a^{i}, t^{j}\right][s, t]\right\rangle .
\end{gathered}
$$

Baumslag and Cleary [Baumslag and Cleary 05] introduced several new families of parafree groups, including some we denote $K_{i, j}$, with $i$ and $j$ relatively prime, presented as

$$
K_{i, j}=\left\langle a, s, t \mid a^{i}[s, a]=t^{j}\right\rangle .
$$

All of these groups have lower central series which are isomorphic to the corresponding quotients for the free group on two generators.

\subsection{Distinguishing Parafree Groups}

A natural question when considering various families of parafree groups is whether or not the groups in these families are in fact distinct. That is, we consider the isomorphism problem for groups in the restricted environment of these families of parafree groups.

There has been some success distinguishing some of these parafree groups theoretically. Fine, Rosenberger, and Stille [Fine et al. 97] were able to solve the isomorphism problem for the family of parafree groups introduced by Baumslag [Baumslag 69] $\langle a, b, t| a^{-1}=$ $\left.\left[b^{i}, a\right]\left[b^{j}, t\right]\right\rangle$ in the case when $j=1$ by taking advantage of the fact that these groups can be regarded as HNN extensions with a single stable letter and a finitely generated base group. By using a version of Nielsen theory 
for HNN extensions, they were able to show that in these groups $G_{i, 1} \not G_{1,1}$ for all $i>1$ and if $i, k$ are primes then $G_{i, 1} \cong G_{k, 1}$ if and only if $i=k$. However, in the general case where neither $i$ nor $j$ is one, these groups do not decompose in the same way and their approach no longer works.

Theoretical efforts to distinguish these groups have been unsuccessful, so we turn to computational efforts. One computational approach to distinguish general finitely presented groups, used effectively by Holt and Rees [Holt and Rees 92] in testisom, is the method of enumerating homomorphisms to fixed finite groups. If two groups are isomorphic, they have the same number of homomorphisms to a fixed finite group. If they have a different number of homomorphisms, the groups must be distinct. Note that such enumeration strategies can only show that groups are distinct; in many cases involving parafree groups, the number of homomorphisms to a target group may be the same as the number of homomorphisms from the corresponding free group which they so closely resemble, as described in [Baumslag and Cleary 05].

Lewis and Liriano [Lewis and Liriano 94] distinguished a number of parafree groups in the class $G_{i, j}$ presented above. They enumerated homomorphisms between $G_{i, j}$ and the finite groups $S L(2, \mathbb{Z} / 4)$ of order 48 and $S L(2, \mathbb{Z} / 5)$ of order 120 for some combinations of $i$ and $j$ both less than 7 and were able to distinguish 10 different isomorphism classes of groups among those. We count epimorphisms onto a selection of simple groups and study a wider collection of parafree examples and succeed in distinguishing all of the ones that we consider.

The net result of these computations is the following theorem:

Theorem 2.1. The 254 distinct parafree groups in the three families $G_{i, j}, H_{i, j}$, and $K_{i, j}$ with $1 \leq i, j \leq 10$ are distinguished from each other by counting epimorphisms onto small simple groups.

This suggests that all of the distinct groups in the families $G_{i, j}, H_{i, j}$, and $K_{i, j}$ might be distinguished by counting the number of epimorphisms onto finite simple groups. Even in the case where the target groups, the finite simple groups, are of small order, such counts lie outside the scope of any computational approach for general families of groups. It may well be that all of the groups discussed here, or indeed that all finitely generated parafree groups, are hyperbolic. In this case the work of Sela [Sela 95] applies; that is to say there would be an algorithm which determines whether or not any pair of the groups above are isomorphic. However the mere existence of such an algorithm still leaves unresolved the question as to which of them are isomorphic. So new tools need to be developed in order to solve this problem.

\subsection{Finite Factor Groups and Equations in Free Groups}

Now suppose that $G$ is a finitely generated group and $T$ is a finite group. The object of this subsection is to record some simple observations about: the set of epimorphisms of the group $G$ onto $T$; the finite factor groups of $G$; and equations in free and relatively free groups. We will concentrate here on equations in free groups.

We start by showing that the number of epimorphisms of a group $G$ onto a finite group $T$ is completely determined by the set of normal subgroups of finite index in $G$.

Proposition 2.2. Let $\operatorname{Epi}(G, T)$ denote the set of epimorphisms from a group $G$ onto a group $T$. Suppose that $G$ and $H$ are finitely generated groups and that $T$ is a finite group. If $G$ and $H$ have the same set of finite images, then

$$
|\operatorname{Epi}(G, T)|=|\operatorname{Epi}(H, T)| .
$$

Proof: Let $\mathbb{V}$ be the variety generated by $T$. By a theorem of B. H. Neumann [Neumann 37] the finitely generated groups in $\mathbb{V}$ are finite. Now every epimorphism, from $G$ onto $T$, factors through the finite group $G / V(G)$, where $V(G)$ is the verbal subgroup of $G$ defined by the variety $\mathbb{V}$. It follows that the number of epimorphisms from $G$ onto $T$ is the number of epimorphisms from $G / V(G)$ onto $T$. Similarly the number of epimorphisms from $H$ onto $T$ is the number of epimorphisms from $H / V(H)$ onto $T$. But $G / V(G) \cong H / V(H)$, which implies that the number of epimorphisms of $G$ onto $T$ is equal to the number of epimorphisms of $H$ onto $T$, as required.

The other observation is contained in the next proposition, the proof of which is obvious.

Proposition 2.3. Let $H$ be a group given by the finite presentation

$$
\begin{aligned}
H=\left\langle a_{1}, \ldots, a_{m}\right| r_{1}\left(a_{1}, \ldots, a_{m}\right) & =1, \ldots, \\
& \left.r_{n}\left(a_{1}, \ldots, a_{m}\right)=1\right\rangle .
\end{aligned}
$$

Suppose that $d=m-n>1$ and that the equations

$$
r_{i}\left(x_{1}, \ldots, x_{m}\right)=1,(i=1, \ldots, n)
$$

hold in a free group $F$. Furthermore, suppose that there exists a $(d-1)$-generator group which is not a quotient 
of $H$. Then the subgroup of $F$ generated by the solutions in $F$ of the system of equations given by (2-1) has rank at most $d-2$.

In order to make use of Proposition 2.3, we need to find examples of finite groups which are not homomorphic images of given finitely presented groups. Even if we have unlimited computational resources, such an approach is not always feasible. Here we proceed theoretically, giving an example of a finite $p$-group which is not a quotient of the group $H=\left\langle a, b, c \mid a^{p} b^{p} c^{p}\right\rangle$. So it follows from Proposition 2.3 that if $a, b$, and $c$ are elements of a free group and if $a^{p} b^{p} c^{p}=1$, then the subgroup generated by $a, b$, and $c$ is abelian. This was proved for $p=2$ in [Lyndon 59]; see also [Baumslag 60] and [Lyndon and Schützenberger 62].

We remark that the existence of nontrivial solutions of equations in nonabelian free groups plays a part in work on the Tarski problem; see, for example, [Sela 01, Sela 03] and [Kharlampovich and Myasnikov 98].

Proposition 2.4. Let $p$ be any given prime. Then there exists a two-generator, finite p-group $G$ which is not a quotient of $H=\left\langle a, b, c \mid a^{p} b^{p} c^{p}\right\rangle$.

We need the following lemma, which is implicit in [Baumslag 68a].

Lemma 2.5. Let $S=A \curlywedge B$ be the wreath product of two groups, $A=\left\langle x \mid x^{p}=1\right\rangle$ and $B=\left\langle y \mid y^{p}=1\right\rangle$, with order the prime $p$. If $z$ is any element in the derived group of $S$, then $z x^{i} y^{j}$ has order $p^{2}$, provided only that $0<i, j<p$.

Proof: Notice that $S$ can be presented in the form

$$
\begin{array}{r}
S=\langle x, y| x^{p}=y^{p}=1,\left[x^{y^{m}}, x^{y^{n}}\right]=1 \\
\text { where } 0 \leq m, n<p\rangle .
\end{array}
$$

It follows that if $S^{\prime}$ denotes the derived group of $S$, then $S / S^{\prime}$ is the direct product of two groups of order $p$ generated by $x S^{\prime}$ and $y S^{\prime}$. Notice also that if $r$ and $s$ are any integers not divisible by $p$, then the mapping which sends $x$ to $x^{r}$ and $y$ to $y^{s}$ can be extended to an automorphism of $S$. It follows that we can assume without loss of generality that $i=1=j$.

Now let $x_{k}=y^{k} x y^{-k}$ for $k=0, \ldots, p-1$. Then every element in $S$ can be expressed in the form

$$
x_{0}^{i_{0}} \ldots x_{p-1}^{i_{p-1}} y^{\ell} \quad \text { where } 0 \leq i_{j} \leq p-1 .
$$

Observe that modulo $S^{\prime}$, the elements $x_{i}$ all coincide. Hence $z \in S^{\prime}$ if and only if it has the form

$$
z=x_{0}^{i_{0}} \ldots x_{p-1}^{i_{p-1}}
$$

where

$$
i_{0}+i_{1}+\ldots+i_{p-1} \equiv 0 \bmod p .
$$

This implies that

$$
z z^{y} \ldots z^{y^{p-1}}=1
$$

Consequently

$$
\begin{aligned}
(z x y)^{p} & =z z^{y} \cdots z^{y^{p-1}} x x^{y} \cdots x^{y^{p-1}} y^{p} \\
& =x_{0} x_{1} \cdots x_{p-1} \neq 1 .
\end{aligned}
$$

So $z x y$ has order $p^{2}$ as claimed.

Proof of Proposition 2.4: We adopt the notation of Lemma 2.5 throughout. Observe that $S$ satisfies the metabelian law (i.e., all commutators commute), the law that the $p^{\text {th }}$-power of all commutators is equal to 1 , the law $x^{p^{2}}=1$, and the law that $S$ is nilpotent with class $p$. Let $G$ be the relatively free group of rank 2 in the variety generated by $S$. It follows that $G$ is metabelian and that $G$ is of exponent $p^{2}$. Since $S$ is a quotient of $G$ it follows that $G$ contains elements of order $p^{2}$. Consequently $G / G^{\prime}$ is a direct product of two cyclic groups of order $p^{2}$.

Our objective is to prove that $G$ is not a quotient of $H=\left\langle a, b, c \mid a^{p} b^{p} c^{p}\right\rangle$. Suppose the contrary. Let $\phi$ be a homomorphism of $H$ onto $G$. The factor group $G / \Phi(G)$ of $G$ by the Frattini subgroup $\Phi(G)$ of $G$, viewed as a vector space over the field of $p$ elements, has dimension two. Since, modulo $\Phi(G)$, the elements $a \phi, b \phi$, and $c \phi$ span this vector space, it follows, renaming the generators if necessary, that $a \phi \Phi(G)$ and $b \phi \Phi(G)$ span $G / \Phi(G)$; consequently $\underline{a}=a \phi$ and $\underline{b}=b \phi$ generate $G$. Hence they freely generate $G$. Consequently the map which sends $\underline{a}$ to $x$ and $\underline{b}$ to $y$ can be extended to a homomorphism $\theta$ from $G$ onto $S$. Let $\underline{c}=c \phi$. Since $\underline{a}$ and $\underline{b}$ generate $G$,

$$
\underline{c}=\underline{a}^{i} \underline{b}^{j} d^{p} e
$$

where $0 \leq i, j<p, d \in G$ and $e$ is contained in the derived group of $G$. Now

$$
\underline{a}^{p} \underline{b}^{p} \underline{c}^{p}=1
$$

It follows that

$$
\underline{c}^{-p}=\underline{a}^{p} \underline{b}^{p} .
$$

Since $G / G^{\prime}$ is the direct product of the cyclic groups $\operatorname{gp}\left(\underline{a} G^{\prime}\right)$ and $\operatorname{gp}\left(\underline{b} G^{\prime}\right)$ of order $p^{2}$, it follows from $(2-2)$ and (2-3), on working modulo $G^{\prime}$, that

$$
i p \equiv p \bmod p^{2}, j p \equiv p \bmod p^{2} .
$$


Hence $i=1+p i^{\prime}, j=1+p j^{\prime}$ for some choice of the integers $i^{\prime}$ and $j^{\prime}$. Consequently $\underline{c}=\underline{a} \underline{b} d^{p} f$, where $d \in G$ and $f \in G^{\prime}$. Now $S$ is generated by elements of order $p$. So the $p^{\text {th }}$ power of an element of $S$ is contained in its derived group. It follows that

$$
1=\left(a^{p} b^{p} c^{p}\right) \phi \theta=x^{p} y^{p}(x y z)^{p}=(x y z)^{p}
$$

where $z \in S^{\prime}$. From Lemma 2.5, xyz has order $p^{2}$ and hence $(x y z)^{p} \neq 1$, which contradicts $(2-4)$. This contradiction implies that $G$ is not a quotient of $H=\langle a, b, c|$ $\left.a^{p} b^{p} c^{p}\right\rangle$, as claimed.

We have verified Proposition 2.4 for $p=2$ and $p=3$ by explicit computation along the lines described in Section 3. Proposition 2.4 gives that the group

$$
\left\langle u, v \mid u^{4}, v^{4},[u, v]^{2},[[u, v], v],[[v, u], u]\right\rangle
$$

with order 32 is not a quotient of $\left\langle a, b, c \mid a^{2} b^{2} c^{2}\right\rangle$ and that the group

$$
\left\langle u, v \mid u^{9}, v^{9},[u, v]^{3},[[[v, u], v], u],[[[v, u], v], v],[[[v, u], u], u]\right\rangle
$$

with order $3^{7}$ is not a quotient of $\left\langle a, b, c \mid a^{3} b^{3} c^{3}\right\rangle$. In general we have that a group with order $p^{p(p-1) / 2+4}$ is not a quotient. By checking all small groups, we have found the smallest nonquotients for $p=2$ and $p=3$, which are unique up to isomorphism, as follows. The group $\left\langle u, v \mid u^{3}, u v u v^{-2}\right\rangle$ with order 24 is not a quotient of $\left\langle a, b, c \mid a^{2} b^{2} c^{2}\right\rangle$. The group

$$
\left\langle u, v \mid u^{9},\left[u^{3}, v\right],\left[u, v^{3}\right],\left[v^{-1}, u^{-1}\right] v^{3}\left[v, u^{-1}\right],\left(u^{-1} v^{-1}\right)^{3}(u v)^{3}\right\rangle
$$

with order $3^{6}$ is not a quotient of $\left\langle a, b, c \mid a^{3} b^{3} c^{3}\right\rangle$; this smaller nonquotient is a quotient of the group provided by Proposition 2.4 .

\section{EXPERIMENTAL DESIGN}

We use Proposition 2.2 to distinguish the parafree groups of Theorem 2.1. The availability of packages for computational group theory, including GAP [The GAP Group 03], Magma [Bosma et al. 97], Magnus [NY Group Theory Cooperative 04], and testisom [Holt and Rees 92] makes it quite easy to experiment with groups. We use GAP, Magma, and components of testisom to study epimorphisms from the groups in question to various finite groups. This kind of computer approach has been used to distinguish infinite groups by Havas and Kovács [Havas and Kovács 84], by Holt and Rees [Holt and Rees 92], and by Lewis and Liriano [Lewis and Liriano 94]. We choose to examine (mainly) simple groups as images and use Atlas notation [Conway et al. 85] for names of these groups.

We constructed tables which give epimorphism counts mainly obtained using straightforward GAP programs. The counts are up to automorphisms of the image group as obtained using the GQuotients command in GAP. (The groups $K_{i, 1} \cong F_{2}$ and are included in the tables for convenient reference.) Various conjectures arise readily from observation of identical rows or columns or other patterns in the tables.

Computations have been done for $G_{i, j}, H_{i, j}$, and $K_{i, j}$ for all relevant $1 \leq i, j \leq 10$ and for epimorphisms to $L_{2}(q)$ for $q \in[5,7,8,9,11]$, plus a few other groups in selected difficult cases. In view of the time taken for some calculations, counts to some larger groups have been done with standalone programs using testisom.

\section{COMPUTATIONAL RESULTS}

In this section we describe the computational results about $G_{i, j}, H_{i, j}$, and $K_{i, j}$ which, inter alia, imply that Theorem 2.1 holds. For $G_{i, j}$ with $1 \leq i, j \leq 10$ all groups have 4 epimorphisms to $L_{2}(3)$ (which is consistent with the results of Lewis and Liriano). A sample table showing epimorphism counts is given in Table 1.

We have similar tables for $G_{i, j}, H_{i, j}$, and $K_{i, j}$ for all relevant $1 \leq i, j \leq 10$ giving epimorphism counts to $L_{2}(q)$ for $q \in[5,7,8,9,11]$. For brevity we omit them here, but they are all available at [Baumslag et al. 04] and at http://www.expmath.org/expmath/volumes/13/13.4/ BaumslagEtAl/tables.pdf.

For $H_{i, j}$ with $1 \leq i, j \leq 10$ all groups (as for $G_{i, j}$ ) have 4 epimorphisms to $L_{2}(3)$. For $K_{i, j}$ with $1 \leq i, j \leq 10$ and

\begin{tabular}{r|rrrrcrrrrr}
\multicolumn{10}{c}{ Epimorphisms : $G_{i, j} \mapsto L_{2}(5)$} \\
\hline \multirow{1}{*}{$i$} & 1 & 2 & 3 & 4 & 5 & 6 & 7 & 8 & 9 & 10 \\
\cline { 2 - 12 } 1 & 16 & 32 & 13 & 32 & 26 & 9 & 36 & 12 & 33 & 22 \\
2 & 12 & 12 & 29 & 32 & 22 & 9 & 12 & 32 & 29 & 22 \\
3 & 33 & 29 & 13 & 9 & 23 & 29 & 33 & 9 & 13 & 19 \\
4 & 32 & 12 & 29 & 12 & 22 & 29 & 12 & 32 & 9 & 22 \\
5 & 26 & 22 & 23 & 22 & 26 & 19 & 26 & 22 & 23 & 22 \\
6 & 9 & 29 & 9 & 29 & 19 & 9 & 29 & 9 & 29 & 19 \\
7 & 16 & 12 & 33 & 32 & 26 & 9 & 16 & 32 & 33 & 22 \\
8 & 32 & 32 & 9 & 12 & 22 & 29 & 32 & 12 & 9 & 22 \\
9 & 33 & 9 & 33 & 9 & 23 & 29 & 13 & 29 & 13 & 19 \\
10 & 22 & 22 & 19 & 22 & 22 & 19 & 22 & 22 & 19 & 22 \\
& & & & & & & & &
\end{tabular}




\begin{tabular}{rrrrr|r}
\multicolumn{7}{c}{ Epimorphism counts to $L_{2}(q)$} \\
\hline$q=5$ & $q=7$ & $q=8$ & $q=9$ & $q=11$ & Group \\
\hline 12 & 60 & 106 & 75 & 307 & $G_{2,7}$ \\
12 & 60 & 120 & 92 & 301 & $G_{1,8}$ \\
12 & 60 & 120 & 92 & 325 & $G_{4,2}$ \\
12 & 60 & 120 & 92 & 325 & $G_{8,4}$ \\
12 & 60 & 120 & 100 & 301 & $G_{2,1}$ \\
12 & 60 & 120 & 100 & 301 & $H_{1,1}$ \\
12 & 60 & 178 & 74 & 350 & $G_{4,7}$ \\
12 & 60 & 192 & 49 & 319 & $G_{4,4}$ \\
12 & 60 & 192 & 49 & 319 & $G_{8,8}$ \\
12 & 68 & 106 & 75 & 307 & $G_{7,2}$ \\
12 & 92 & 192 & 81 & 319 & $G_{2,2}$
\end{tabular}

TABLE 2.

$\operatorname{gcd}(i, j)=1$ most groups (as for $G_{i, j}$ and $H_{i, j}$ ) have 4 epimorphisms to $L_{2}(3)$. However $K_{2,3}, K_{2,9}, K_{4,3}, K_{4,9}$, $K_{8,3}, K_{8,9}, K_{10,3}$, and $K_{10,9}$ have 8 , distinguishing them from the other groups.

To study all of these parafree groups we can sort them according to the quintuple of epimorphism counts onto $L_{2}(5), L_{2}(7), L_{2}(8), L_{2}(9)$, and $L_{2}(11)$. For example, the part of the sorted table with 12 epimorphisms onto $L_{2}(5)$ contains 11 groups and is given in Table 2 .

This part of the sorted table shows that the pairs $\left(G_{4,2}, G_{8,4}\right),\left(G_{2,1}, H_{1,1}\right)$, and $\left(G_{4,4}, G_{8,8}\right)$ are not distinguished by these epimorphism counts. However, apart from possible isomorphisms among these pairs, these 11 groups are different from all other $G_{i, j}, H_{i, j}$, and $K_{i, j}$ in our range (and from $F_{2}$ ).

Ignoring the 10 copies of $F_{2}$ provided by $K_{i, 1}$ there are 253 groups. Only 20 pairs (from these 253 plus $F_{2}$ itself) are not distinguished by the epimorphism counts to $L_{2}(q)$ for $q \in[5,7,8,9,11]$. For each of these pairs we list epimorphism counts onto $L_{2}(q)$ for $q \in[13,16,17]$ (see Table 3).

This list leaves us with only two pairs of groups to distinguish: $\left(G_{4,2}, G_{8,4}\right)$ and $\left(G_{2,1}, H_{1,1}\right)$. For these two pairs we list epimorphism counts onto $L_{2}(q)$ for $q \in[19,23,25,27,29,31]$ (see Table 4).

Thus the pair $\left(G_{4,2}, G_{8,4}\right)$ are distinguished by epimorphism counts onto $L_{2}(23), L_{2}(25)$, and $L_{2}(31)$. However the pair $\left(G_{2,1}, H_{1,1}\right)$ are not distinguished by epimorphism counts onto $L_{2}(q)$ for $q \leq 31$. The choice of $L_{2}(q)$ as simple images was somewhat arbitrary, chosen for ease of use.

To differentiate our final pair of groups we study epimorphism counts to all moderately sized simple groups. We counted epimorphisms to each of the 43 nonabelian
Epimorphism counts to $L_{2}(q)$

\begin{tabular}{cccc}
\hline Groups & $q=13$ & $q=16$ & $q=17$ \\
\hline$G_{2,4}, G_{4,8}$ & 476,476 & 736,736 & 1178,1114 \\
$G_{4,2}, G_{8,4}$ & 480,480 & 882,882 & 1276,1276 \\
$G_{4,4}, G_{8,8}$ & 482,482 & 882,882 & 1309,1053 \\
$G_{3,3}, G_{9,9}$ & 548,548 & 975,975 & 946,1162 \\
$G_{1,9}, G_{9,1}$ & 530,616 & 965,829 & 1196,1196 \\
$G_{3,5}, G_{5,3}$ & 616,478 & 955,1151 & 1157,1013 \\
$G_{3,7}, G_{7,3}$ & 529,551 & 1101,1237 & 1262,1226 \\
$G_{4,5}, G_{5,4}$ & 508,499 & 862,1058 & 1022,1310 \\
$G_{4,6}, G_{6,4}$ & 441,524 & 1047,1047 & 1083,1155 \\
$G_{5,9}, G_{9,5}$ & 457,539 & 1151,1091 & 1176,1312 \\
$G_{10,6}, G_{6,10}$ & 448,556 & 1097,901 & 1109,1253 \\
$G_{10,8}, G_{8,10}$ & 469,466 & 1058,862 & 1182,1046 \\
$K_{1,4}, K_{1,8}$ & 459,459 & 936,936 & 2001,2543 \\
$K_{3,4}, K_{3,8}$ & 602,602 & 1041,1041 & 1889,2463 \\
$K_{5,4}, K_{5,8}$ & 561,561 & 990,990 & 1319,1115 \\
$K_{7,4}, K_{7,8}$ & 585,585 & 936,936 & 2340,3896 \\
$K_{9,4}, K_{9,8}$ & 602,602 & 941,941 & 2183,3167 \\
$K_{2,3}, K_{2,9}$ & 639,639 & 939,939 & 1720,2155 \\
$G_{2,1}, H_{1,1}$ & 522,522 & 882,882 & 1284,1284 \\
$G_{6,5}, H_{1,5}$ & 500,478 & 1173,765 & 1198,971
\end{tabular}

TABLE 3

\begin{tabular}{r|rr|rr}
\multicolumn{5}{c}{ Epimorphism counts to $L_{2}(q)$} \\
\hline$q$ & $G_{4,2}$ & $G_{8,4}$ & $G_{2,1}$ & $H_{1,1}$ \\
\hline 19 & 1818 & 1818 & 1708 & 1708 \\
23 & 3088 & 3064 & 3100 & 3100 \\
25 & 1748 & 1616 & 1765 & 1765 \\
27 & 1935 & 1935 & 1949 & 1949 \\
29 & 5704 & 5704 & 5802 & 5802 \\
31 & 7186 & 7314 & 7394 & 7394
\end{tabular}

TABLE 4.

simple groups with order up to 285852 (the order of $\left.L_{2}(83)\right)$. Epimorphism counts reveal that $\left(G_{2,1}, H_{1,1}\right)$ are distinguished by 11 of these 43 simple groups, (but not by any $L_{2}(q)$ with $\left.q \leq 83\right)$. Thus, with simple groups listed in order of increasing size and with counts up to inner automorphisms (since we used the permim command of testisom) we have the results in Table 5 .

(We note that our choice of permim for all of these calculations was for uniformity. For $A_{8}$ and $A_{9}$ we can obtain equivalent results more quickly. Thus, epimorphism counts onto alternating (and symmetric) groups can in general be done much faster by using the Low Index Subgroups algorithm.)

So, by counting epimorphisms onto small simple groups, we deduce that Theorem 2.1 holds. 


\begin{tabular}{r|rr}
\multicolumn{3}{c}{ Epimorphism counts } \\
\hline Image & $G_{2,1}$ & $H_{1,1}$ \\
\hline$L_{3}(3)$ & 5432 & 5288 \\
$U_{3}(3)$ & 5116 & 5338 \\
$M_{11}$ & 7238 & 7334 \\
$A_{8}$ & 18032 & 17808 \\
$L_{3}(4)$ & 16812 & 16140 \\
$U_{4}(2)$ & 24996 & 25230 \\
$U_{3}(4)$ & 58524 & 58588 \\
$M_{12}$ & 90140 & 89948 \\
$U_{3}(5)$ & 125964 & 126192 \\
$J_{1}$ & 174226 & 174274 \\
$A_{9}$ & 169988 & 170062
\end{tabular}

TABLE 5.

No finite set of values can be used to distinguish an infinite family of groups. There are severe limits on any single finite group being used to distinguish one-relator parafree groups (and likewise limits on any finite collection of finite groups). Exemplifying this, in the context of the method for distinguishing groups used here, it is shown in [Baumslag and Cleary 05] that if $T$ is any given finite group then there are infinite families of one-relator parafree groups $\Gamma_{i}$ such that the number of homomorphisms from $\Gamma_{i}$ into $T$ is the same as the number of homomorphisms from the appropriate free group into $T$.

\section{FURTHER QUESTIONS}

We expect that the groups in these families are (essentially) all distinct but we do not yet have the tools for theoretical or experimental proof. However we now have successfully distinguished what might be viewed as a meaningful initial collection. It seems reasonable to conjecture that they are all distinguished by counting epimorphisms onto simple groups.

We expect that the groups in these families are easier to distinguish than more complicated nonfree parafree groups. For example, the groups $H_{i, j}$ considered here are all particular examples of the more general $H_{w}$, where in $H_{i, j}$, the word $w$ has a restricted form and lies in the first derived group. We expect that examples in $H_{w}$ where $w$ is more complicated or lies in the second or highernumbered derived group are more difficult to distinguish from the free groups and from each other. We hope that other computational approaches, such as the enumeration of finite index subgroups or examining kernels of homomorphisms, may effectively distinguish more of these groups.

\section{ACKNOWLEDGMENTS}

This work was supported in part by NSF grants \#02-02382 and \#02-15942 and in part by the Australian Research Council.

\section{REFERENCES}

[Baumslag 60] Gilbert Baumslag. "On a Problem of Lyndon." J. London Math. Soc. 35 (1960), 30-32.

[Baumslag 67a] Gilbert Baumslag. "Groups with the Same Lower Central Sequence as a Relatively Free Group. I. The Groups." Trans. Amer. Math. Soc. 129 (1967), 308321.

[Baumslag 67b] Gilbert Baumslag. "Some Groups that Are Just About Free." Bull. Amer. Math. Soc. 73 (1967), 621-622.

[Baumslag 68a] Gilbert Baumslag. "More Groups that Are Just About Free." Bull. Amer. Math. Soc. 74 (1968), 752-754.

[Baumslag 68b] Gilbert Baumslag. "On the Residual Nilpotence of Certain One-Relator Groups." Comm. Pure Appl. Math. 21 (1968), 491-506.

[Baumslag 69] Gilbert Baumslag. "Groups with the Same Lower Central Sequence as a Relatively Free Group. II. Properties." Trans. Amer. Math. Soc. 142 (1969), 507538.

[Baumslag 94] Gilbert Baumslag. "Musings on Magnus." In The Mathematical Legacy of Wilhelm Magnus: Groups, Geometry and Special Functions (Brooklyn, NY, 1992), pp. 99-106. Providence, RI: Amer. Math. Soc., 1994.

[Baumslag and Cleary 05] Gilbert Baumslag and Sean Cleary. "Parafree One-Relator Groups." To appear in Journal of Group Theory, 2005.

[Baumslag et al. 04] Gilbert Baumslag, Sean Cleary, and George Havas. "Experimenting with Infinite Groups, I: Tables." Available from World Wide Web (http://www.itee.uq.edu.au/ havas/ewig1tables), 2004 .

[Bosma et al. 97] W. Bosma, J. Cannon, and C. Playoust. "The Magma Algebra System I: The User Language." Journal of Symbolic Computation 24 (1997), 235-265. See also http://magma.maths.usyd.edu.au/magma/.

[Conway et al. 85] J. H. Conway, R. T. Curtis, S. P. Norton, R. A. Parker, and R. A. Wilson. Atlas of Finite Groups. Oxford, UK: Oxford University Press, 1985.

[Fine et al. 97] Benjamin Fine, Gerhard Rosenberger, and Michael Stille. "The Isomorphism Problem for a Class of Para-Free Groups." Proc. Edinburgh Math. Soc. (2) 40:3 (1997), 541-549.

[The GAP Group 03] The GAP Group. GAP - Groups, Algorithms, and Programming, Version 4.3. Aachen, St Andrews, 2003. See also http://www.gap-system.org/ 
[Havas and Kovács 84] George Havas and L. G. Kovács. "Distinguishing Eleven Crossing Knots." In Computational Group Theory (Durham, 1982), pp. 367-373. New York: Academic Press, 1984.

[Holt and Rees 92] D. F. Holt and S. Rees. "Testing for Isomorphism between Finitely Presented Groups." In Groups, Combinatorics and Geometry, edited by M. W. Liebeck and J. Saxl, pp. 459-475. Cambridge, UK: Cambridge University Press, 1992.

[Kharlampovich and Myasnikov 98] Olga Kharlampovich and Alexei Myasnikov. "Tarski's Problem About the Elementary Theory of Free Groups Has a Positive Solution." Electron. Res. Announc. Amer. Math. Soc. 4 (electronic) (1998), 101-108.

[Lewis and Liriano 94] Robert H. Lewis and Sal Liriano. "Isomorphism Classes and Derived Series of Certain Almost-Free Groups." Experiment. Math. 3:3 (1994), $255-258$.

[Lyndon 59] R. C. Lyndon. "The Equation $a^{2} b^{2}=c^{2}$ in Free Groups." Michigan Math. J. 6 (1959), 89-95.
[Lyndon and Schützenberger 62] R. C. Lyndon and M. P. Schützenberger. "The Equation $a^{M}=b^{N} c^{P}$ in a Free Group." Michigan Math. J. 9 (1962), 289-298.

[Magnus 30] W. Magnus. "Über diskontinuierliche Gruppen mit einer definierenden Relation. (Der Freiheitssatz.)" $J$. Reine Agnew. Math. 163 (1930), 141-165.

[NY Group Theory Cooperative 04] The New York Group Theory Cooperative. Magnus: A Graphically-Oriented Software System for Computational Group Theory. See http://www.grouptheory.org/, 2004.

[Neumann 37] B.H. Neumann. "Identical Relations in Groups. I." Math. Annalen 114 (1937), 506-528.

[Sela 95] Z. Sela. "The Isomorphism Problem for Hyperbolic Groups. I." Ann. of Math. (2) 141 (1995), 217-283.

[Sela 01] Zlil Sela. "Diophantine Geometry over Groups. I. Makanin-Razborov Diagrams." Publ. Math. Inst. Hautes Études Sci. 93 (2001), 31-105.

[Sela 03] Z. Sela. "Diophantine Geometry over Groups. II. Completions, Closures and Formal Solutions." Israel J. Math. 134 (2003), 173-254.

Gilbert Baumslag, Department of Mathematics, City College of New York, City University of New York, New York, NY 10031 (gilbert@groups.sci.ccny.cuny.edu)

Sean Cleary, Department of Mathematics, City College of New York, City University of New York, New York, NY 10031 (cleary@sci.ccny.cuny.edu)

George Havas, ARC Centre for Complex Systems, School of Information Technology and Electrical Engineering, The University of Queensland, Queensland 4072, Australia (havas@itee.uq.edu.au)

Received May 27, 2004; accepted August 2, 2004. 